\title{
Cinza de biomassa rica em calcário como material carbonático em sistemas cimentícios de base Portland
}

\section{(Calcium-rich biomass ash as a carbonate material in Portland base systems)}

\author{
J. E. F. Nascimento ${ }^{1 *}$, A. C.V.Nóbrega ${ }^{2}$, H. C. Ferreira ${ }^{1}$, G. A. Neves ${ }^{1}$, L. N. L. Santana ${ }^{1}$ \\ ${ }^{1}$ Universidade Federal de Campina Grande, Programa de Pós-graduação em Ciência e Engenharia de Materiais, \\ Campina Grande, PB, Brasil \\ ${ }^{2}$ Universidade Federal de Pernambuco, Programa de Pós-graduação em Engenharia Civil e Ambiental, \\ Campus do Agreste, Caruaru, PE, Brasil
}

\begin{abstract}
Resumo
O uso de materiais alternativos, em substituição ao clínquer, tem sido amplamente estudado, a fim de reduzir custos e minimizar os danos ambientais causados pela produção do cimento Portland. Investigou-se a influência do uso da cinza de algaroba (Prosopis juliflora), material rico em carbonato de cálcio, nos mecanismos de hidratação e formação das principais fases hidratadas do cimento Portland. As características físicas e químicas das matérias-primas foram avaliadas. Composições com 5\%, $10 \%$ e $15 \%$ de cinza de algaroba foram analisadas e comparadas com o cimento Portland comercial com fíler calcário (6-10\%), CPII-F. A cinza de algaroba é um potencial fíler alternativo para aplicação em sistemas cimentícios de base Portland, atuando de forma semelhante ao fíler calcário em termos de nucleação da fase C-S-H, estabilização da etringita e tendência à formação de carboaluminatos.

Palavras-chave: biomassa rica em calcário, algaroba, cinza, hidratação, fíler calcário.
\end{abstract}

\begin{abstract}
The use of alternative materials to substitute clinker has been widely studied to reduce costs, as well as to minimize environmental damage caused by the Portland cement production. It was evaluated the influence of the calcium-rich algaroba (Prosopis juliflora) ash on the Portland cement hydration mechanism, and its main hydrated phases. The physical and chemical characteristics of the raw materials have been evaluated. Compositions containing 5\%, 10\%, and 15\% of algaroba ash were analyzed and compared with commercial Portland cement with limestone filler (6-10\%), CPII-F. The algaroba ash is a potential alternative filler for application in Portland-based cement systems. It acted similarly to the limestone filler regarding the C-S-H nucleation, ettringite stabilization and the tendency to form carboaluminates.
\end{abstract}

Keywords: highly-calcareous biomass, algaroba, ash, hydration, limestone filler.

\section{INTRODUÇÃO}

Os processos de combustão de biomassa para geração de energia e/ou calor produzem cinzas como produto secundário. Em geral, essas cinzas são ricas em compostos de base cálcio, silicosos ou sílico-aluminosos [1-3]. Quando ricas em sílica ou sílica-alumina atuam preferencialmente como materiais pozolânicos $[4,5]$. Por outro lado, quando ricas em cálcio, especificamente na forma de calcário, podem ser capazes de atuar como fíler calcário [6]. O fíler calcário pode ser incorporado nos sistemas de base Portland no início do processo de fabricação do cimento, CPII-F, contendo de $6 \%$ a $10 \%$ de fíler calcário [7] com no mínimo $85 \%$ de carbonato de cálcio em substituição ao clínquer [7-9], ou como carga na fabricação de pastas, argamassas e concretos vibrados e autoadensável [10-16]. O fíler calcário é comum à maioria dos tipos de cimentos Portland produzidos no Brasil e no mundo

*jonatas.nascimento@unifavip.edu.br

(D) https://orcid.org/0000-0002-5850-1866
[7-9, 17-19]. Quando se propõe o uso de um fíler calcário alternativo, há reduções energética, de custo e de emissão de $\mathrm{CO}_{2}$ [20], por substituir parcialmente o volume de clínquer e/ ou cimento Portland nos sistemas cimentícios. Pesquisadores estudaram a adição de materiais alternativos em substituição ao clínquer [10-15, 21, 22], dentre eles o fíler de quartzo, o fíler calcário, a escória de alto forno e cinza volante, nas proporções de $10 \%$ a $50 \%$.

No âmbito da proposta do uso de cinza de biomassa, rica em calcário, como fíler alternativo em sistemas cimentícios de base Portland, encontra-se a cinza de algaroba (Prosopis juliflora) [23]. A algaroba é uma espécie vegetal presente em abundância no nordeste brasileiro. A lenha de algaroba serve como fonte de energia, além do setor têxtil, no setor ceramista. A incorporação de cinza de madeira em materiais cimentícios tem sido foco de vários estudos, atuando positivamente na resistência mecânica, resistência a cloretos, durabilidade de argamassa e concreto, permeabilidade ao ar e carbonatação [1, 24-30]. Além disso, como o fíler calcário usualmente utilizado é extraído de fontes não renováveis, envolvendo 
altos gastos energéticos com extração, britagem e moagem, a redução energética é ainda superior, uma vez que as cinzas de biomassa são produtos gerados em granulometrias reduzidas, dessa forma diminui o gasto energético dos sistemas [12].

As alterações na hidratação do sistema cimentício de base Portland, decorrente da presença dos fíleres calcários usuais, são amplamente estudadas [1, 12, 21, 31, 32]. A cinética e o calor de hidratação nas primeiras horas são alterados, atribuindo-se o fenômeno à nucleação de novas fases de C-S-H na superfície dos finos adicionados, principalmente do fíler calcário [1, 6, 12, 21, 31-34]. Além disso, a formação de fases carboaluminatos é evidenciada na presença do calcário, influenciando positivamente a microestruturas do concreto $[12,35]$. Autores também reportam alterações na formação das fases trissulfato (AFt) e monossulfato (AFm) [1, 12, 32] com a estabilização da fase etringita no decorrer da hidratação, tendo em vista que sua formação e decomposição acontece nas primeiras idades. Após a formação de etringita, quando a quantidade de sulfatos é reduzida no sistema, acontece a sua decomposição em monossulfato. Na presença do fíler calcário, essa decomposição é retardada e há a formação de novas fases, que são os carboaluminatos [32,36,37]. Todavia, o mecanismo de hidratação é complexo e facilmente influenciado pelo efeito do empacotamento, principalmente nas primeiras idades [22, 31, 32]. Nesse cenário, avaliou-se a influência do uso da cinza de algaroba (Prosopis juliflora), material rico em carbonato de cálcio, nos mecanismos de hidratação e formação das principais fases hidratadas do cimento Portland. Os produtos de hidratação foram avaliados por difração de raios $\mathrm{X}$, análise termogravimétrica e microscopia eletrônica de varredura. Para tanto, cimentos foram produzidos em laboratório (clínquer+gesso) com substituição do clínquer pela cinza de algaroba em até $15 \%$ em massa e seus comportamentos comparados com o cimento laboratorial sem adições, bem como com o cimento comercial brasileiro CPII-F [17].

\section{MATERIAIS E MÉTODOS}

O clínquer $\left(0,87 \mathrm{~m}^{2} / \mathrm{g}, 3,04 \mathrm{~g} / \mathrm{cm}^{3}\right)$ e o cimento CPII-F foram disponibilizados pela empresa Nassau, adquiridos em sacos de $50 \mathrm{~kg}$ e devidamente condicionados. Foi necessário formular o CPI (sem adições) em laboratório, em função da ausência comercial no Brasil. Para formulação do cimento CPI, o clínquer foi submetido à moagem em moinho rotativo de alta energia PM-100 (Retsch), por 6 min a 350 rpm; após esse período, o material foi classificado granulometricamente através de peneira ABNT n $200(0,074 \mathrm{~mm})$. Em seguida, foram adicionados $5 \%$ de gesso $\left(2,85 \mathrm{~m}^{2} / \mathrm{g}, 2,53 \mathrm{~g} / \mathrm{cm}^{3}, 55,63 \%\right.$ $\mathrm{SO}_{3}, 41,05 \% \mathrm{CaO}, 2,37 \% \mathrm{P}_{2} \mathrm{O}_{3}$ ), fornecido pela indústria Chaves. A cinza de algaroba foi cedida pela lavanderia Nova Geração, Caruaru-PE, que utilizou a madeira da algaroba (Prosopis juliflora) como única fonte de biomassa em sua linha de produção. A cinza leve foi coletada do interior dos fornos de queima da biomassa e submetida ao peneiramento na malha $\mathrm{ABNT} \mathrm{n}^{\circ} 200(0,074 \mathrm{~mm})$.

Para as caracterizações físico-químicas das matériasprimas, foram utilizados os métodos: a) área superficial específica por BET (Micromeritics, Nova 3200), com prétratamento das amostras a $60^{\circ} \mathrm{C}$ e pressão de $100 \mu \mathrm{mHg}$ por $24 \mathrm{~h}$; b) densidade real por picnometria de gás hélio (Quantachrome, Upyc 1200e v5. 04 Pycnometer); c) análise granulométrica à laser (Cilas, mod. 1064); d) análise termogravimétrica, TG (TA Instruments, SDT-Q600 Simultaneous TG), em atmosfera de nitrogênio, com fluxo de $20 \mathrm{~mL} \cdot \mathrm{min}^{-1}$, cadinho de alumina, taxa de aquecimento de $10{ }^{\circ} \mathrm{C} \cdot \mathrm{min}^{-1}$ e faixa de temperatura entre a ambiente e $1000{ }^{\circ} \mathrm{C}$; e) difração de raios $\mathrm{X}, \mathrm{DRX}$ (Shimadzu, XRD 6000), sendo a radiação utilizada $\mathrm{K} \alpha$ do $\mathrm{Cu}$, tensão de $40 \mathrm{kV}$, corrente de $30 \mathrm{~mA}$, varredura de $5^{\circ}$ até $60^{\circ}$ e velocidade do goniômetro de $2 \% \mathrm{~min}$; f) fluorescência de raios X com EDX (Shimadzu, EDX 720); e g) microscopia eletrônica de varredura (Shimadzu, SSX-550). A cinza de algaroba apresentou predominância de compostos com cálcio, $\mathrm{CaO}(77,31 \%), \mathrm{SiO}_{2}(3,39 \%), \mathrm{Al}_{2} \mathrm{O}_{3}(1,55 \%), \mathrm{K}_{2} \mathrm{O}(8,57 \%)$ e $4,08 \%$ de outros óxidos. Segundo a equação [ $\left.\left(\mathrm{Na}_{2} \mathrm{O}\right) \mathrm{e}\right]=$ $\left[\mathrm{Na}_{2} \mathrm{O}\right]+0,64 .\left[\mathrm{K}_{2} \mathrm{O}\right]$, que determina um teor de álcalis acima de $1,5 \%$ [38], observou-se que para as adições estudadas o teor de álcalis foi dentro do padrão.

Para preparação dos cimentos com adição, as cinzas de algaroba e o cimento CPI foram homogeneizados em misturador hélice por 3 min em baixa rotação e denominados de C0 (sem adição), bem como CC5, CC10 e CC15 para adições de 5\%, 10\% e $15 \%$ de cinza de algaroba (Tabela I). As pastas estudadas foram produzidas com fator água/cimento de 0,5 [12, 39-41] em argamassadeira planetária com cuba de capacidade de 3 L. A escolha dessa relação foi definida por ser suficiente para garantir a plena hidratação dos sistemas [12,3941]. Cimento e água foram adicionados à cuba, nessa ordem, e posteriormente misturados em velocidade baixa por $1 \mathrm{~min}$, tempo determinado para a molhagem visual dos pós, seguida de mais 2 min em velocidade alta. A moldagem foi realizada em moldes circulares de $20 \mathrm{~mm}$ de diâmetro por $20 \mathrm{~mm}$ de altura [42, 43]. Após o período de $24 \mathrm{~h}$, os corpos de provas foram desmoldados e curados em câmara úmida pelo período de 7 e 28 dias. O processo de hidratação foi interrompido por meio de imersão em acetona por $1 \mathrm{~h}$, seguido de secagem em estufa a $50{ }^{\circ} \mathrm{C}$ por 24 h; na sequência, os corpos de prova foram moídos e peneirados em malha ABNT no $200(0,074 \mathrm{~mm})$ para realização das caracterizações mineralógicas por DRX e termogravimétricas.

Tabela I - Composições dos cimentos estudados. [Table I - Studied cements compositions.]

\begin{tabular}{cccccc}
\hline Amostra & C0 & CC5 & CC10 & CC15 & CPII-F \\
\hline Cimento (\%) & 100 & 95 & 90 & 85 & 100 \\
Cinza (\%) & 0 & 5 & 10 & 15 & 0 \\
\hline
\end{tabular}

\section{RESULTADOS E DISCUSSÃO}

Os resultados da análise química dos cimentos formulados e do CPII-F comercial encontram-se na Tabela II. Verificaram-se concentrações menores dos elementos silício e alumínio comparativamente ao CPII-F, como também 
Tabela II - Composição química (\%) dos cimentos (clínquer+5\% gesso).

[Table II - Chemical composition (\%) of cements (clinker $+5 \%$ gypsum).]

\begin{tabular}{ccccccccccccccc}
\hline Amostra & $\mathrm{CaO}$ & $\mathrm{SiO}_{2}$ & $\mathrm{Al}_{2} \mathrm{O}_{3}$ & $\mathrm{Fe}_{2} \mathrm{O}_{3}$ & $\mathrm{SO}_{3}$ & $\mathrm{MgO}$ & $\mathrm{K}_{2} \mathrm{O}$ & $\mathrm{TiO}_{2}$ & $\mathrm{BaO}$ & $\mathrm{SrO}$ & $\mathrm{MnO}$ & $\mathrm{NbO}$ & $\mathrm{ZrO}_{2}$ & $\mathrm{P}_{2} \mathrm{O}_{5}$ \\
\hline CPII-F & 61,27 & 20,12 & 6,27 & 4,86 & 3,90 & 1,47 & 1,14 & 0,47 & 0,27 & 0,09 & 0,08 & 0,04 & 0,03 & - \\
$\mathrm{C} 0$ & 68,69 & 13,51 & 2,95 & 2,67 & 7,91 & 2,75 & 1,21 & 0,19 & - & 0,06 & 0,02 & - & 0,06 & 1,39 \\
$\mathrm{CC} 5$ & 69,68 & 13,07 & 2,92 & 2,59 & 5,29 & 2,87 & 1,83 & 0,19 & - & 0,09 & 0,02 & - & 0,07 & 1,46 \\
$\mathrm{CC} 10$ & 70,09 & 11,81 & 2,62 & 2,46 & 5,84 & 2,94 & 2,31 & 0,17 & - & 0,10 & 0,04 & - & 0,02 & 1,59 \\
$\mathrm{CC} 15$ & 70,10 & 13,50 & 2,92 & 2,67 & 5,96 & 3,00 & 1,61 & 0,17 & - & 0,07 & 0,01 & - & 0,06 & 1,41 \\
Cinza & 77,31 & 3,39 & 1,55 & 0,45 & 0,76 & 2,69 & 8,57 & - & - & 0,18 & - & - & - & - \\
\hline
\end{tabular}

valores superiores de cálcio. Essas alterações possivelmente foram causadas pelas diferentes características químicas dos clínqueres, das matérias-primas utilizadas e das condições de moagem [44]. A variação dos elementos cálcio e silício no cimento interfere diretamente nas fases anidras $\mathrm{C}_{3} \mathrm{~S}$ e $\mathrm{C}_{2} \mathrm{~S}$, nas quais uma maior concentração de cálcio leva a uma produção preponderante de alita, principal responsável pelas fases hidratadas $\mathrm{C}-\mathrm{S}-\mathrm{H} \mathrm{e} \mathrm{Ca}(\mathrm{OH})_{2}$ [45]. Além disso, a relação $\mathrm{Ca} / \mathrm{Si}$ também tem influência na composição química e microestrutural do C-S-H, prolongando suas cadeias com elevação da sílica e/ou a maior difusão de cálcio ou álcalis interlamelares na elevação do cálcio [46, 47].

A cinza leve foi coletada do interior dos fornos de queima da biomassa e submetido ao peneiramento na malha ABNT $\mathrm{n}^{\circ} 200(0,074 \mathrm{~mm})$, devidamente acondicionada e ensaiada por DRX. As fases cristalinas $\mathrm{CaCO}_{3}$ (JCPDS 72-1937), $\mathrm{CaSO}_{3}$ (JCPDS 84-0962) e $\mathrm{K}_{2} \mathrm{CO}_{3}$ (JCPDS 870730) foram identificadas na cinza de algaroba, conforme difratograma exposto na Fig. 1. A presença de carbonatos de cálcio e potássio foi proveniente das condições de combustão da madeira e oxidações pós-queima, como também decorrente da composição química da cinza. Essa composição é diretamente influenciada pelas características de crescimento da algaroba e à disponibilidade de macronutrientes no solo, como $\mathrm{Ca}, \mathrm{Si}, \mathrm{K}$ e N. Um composto químico inerte como o carbonato de cálcio é facilmente adicionado em produtos cimentícios [48]. A ausência de óxido de cálcio e de quantidades expressivas de outros tipos de óxidos no difratograma da cinza é positivo, uma vez que os óxidos, compostos químicos expansivos após contato com a água, possibilitariam reações de expansão tardias no sistema. Os processos expansivos caracterizam um problema do ponto de vista dos sistemas cimentícios, pois a falta de conhecimento das características e tempo de hidratação desses compostos poderiam levar à formação de microtrincas à medida que a hidratação evoluísse, reduzindo assim significativamente algumas propriedades no estado endurecido: resistência à compressão, resistência à tração, permeabilidade e durabilidade [49]. A Fig. 2 ilustra as curvas termogravimétricas TG e DTG da cinza de algaroba. Verificaram-se perdas de massa de $0,34 \%$ e $0,27 \%$ ente 347 e $415{ }^{\circ} \mathrm{C}$, referentes ao hidróxido de potássio e de cálcio. A presença dos carbonatos (de cálcio e de potássio) foi relacionada à perda de massa na ordem de $31,07 \%$ a $801{ }^{\circ} \mathrm{C}$.

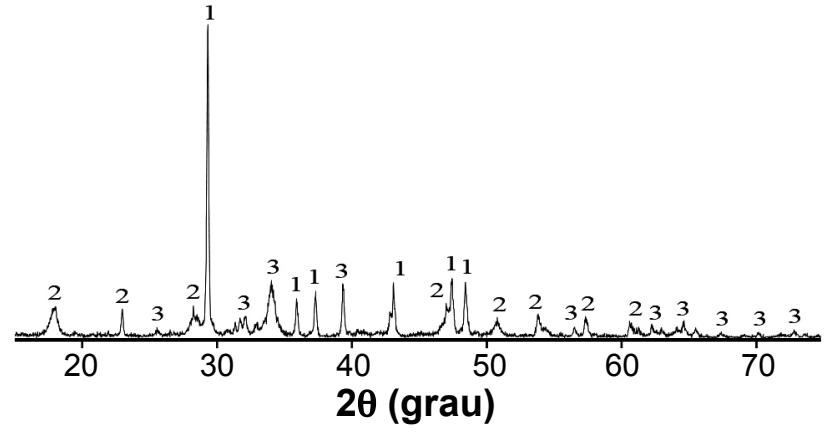

Figura 1: Difratograma de raios $\mathrm{X}$ da cinza de algaroba: $1-\mathrm{CaCO}_{3}$; $2-\mathrm{CaSO}_{3} ;$ e $3-\mathrm{K}_{2} \mathrm{CO}_{3}$.

[Figure 1: XRD pattern of algaroba ash.]

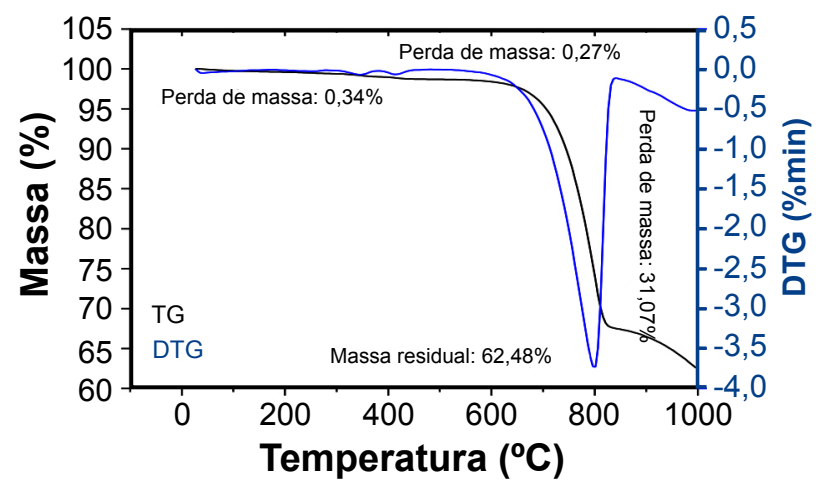

Figura 2: Curvas termogravimétricas TG e DTG da cinza de algaroba.

[Figure 2: TG and DTG thermogravimetric curves of the algaroba ash.]

As principais fases do cimento Portland foram identificadas para todos os cimentos estudados $(\mathrm{C} 0, \mathrm{CC} 5, \mathrm{CC} 10, \mathrm{CC} 15$, CPII-F), Fig. 3: C C S (JCPDS 49-0442), C S (JCPDS 33-0302), $\mathrm{C}_{3} \mathrm{~A}$ (JCPDS 038-1429), $\mathrm{C}_{4} \mathrm{AF}$ (JCPDS 30-0226) e $\mathrm{CaCO}_{3}$ (JCPDS 72-1937). Picos mais preponderantes de carbonato de cálcio, $\mathrm{CaCO}_{3}$, e de ferroaluminato tetracálcio, $\mathrm{C}_{4} \mathrm{AF}$, estão diretamente relacionados com a composição química do clínquer, adições ao cimento Portland e as condições de moagem no processo de produção [17]. Verificou-se que os cimentos preparados em laboratório não apresentaram alterações significativas nos compostos anidros, quando comparados ao cimento Portland comercial CPII-F. Apesar das composições químicas discrepantes do clínquer processado laboratorialmente e comercial usado na produção do CPII-F, 
Tabela II, as principais fases anidras foram identificadas nos cimentos estudados.

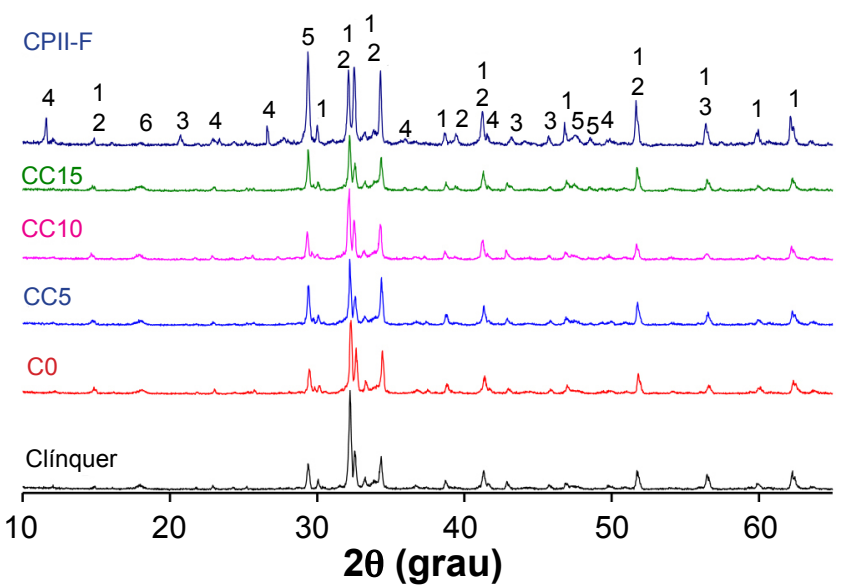

Figura 3: Difratogramas de raios $\mathrm{X}$ dos cimentos estudados: $1-\mathrm{C}_{3} \mathrm{~S}$; $2-\mathrm{C}_{2} \mathrm{~S} ; 3-\mathrm{C}_{3} \mathrm{~A} ; 4-\mathrm{C}_{4} \mathrm{AF} ; 5-\mathrm{CaCO}_{3}$; e 6-CaSO $\mathrm{Ca}_{4} .1 / 2 \mathrm{H}_{2} \mathrm{O}$.

[Figure 3: XRD patterns of studied cements.]

A Fig. 4 ilustra as curvas de distribuição granulométrica dos cimentos produzidos laboratorialmente (clínquer+gesso+cinza), cinza de algaroba e CPII-F comercial. Na Tabela III encontram-se ainda expostas as faixas de distribuição granulométrica nas regiões D10, D50 e D90, a área superficial e os valores de densidade real de todos os cimentos avaliados. A cinza de algaroba apresentou tanto diâmetro médio como D90 próximos aos do CPII-F (Tabela III), favorecendo assim a redução do diâmetro médio dos cimentos formulados com o aumento dessa adição. $\mathrm{O}$ maior valor de área superficial específica da cinza de algaroba $\left(4,400 \mathrm{~m}^{2} / \mathrm{g}\right)$ beneficia também uma melhor interação física com o cimento Portland. Sua menor densidade real $\left(2,552 \mathrm{~g} / \mathrm{cm}^{3}\right)$ favorece a produção de concretos e argamassas mais leves. A inclusão de materiais finamente moídos influencia a reologia do concreto, reduz o processo de exsudação superficial ou interna, aprimorando as condições de trabalhabilidade e resistência mecânica final. A granulometria refinada da cinza de algaroba, bem como sua maior área superficial, comparativamente às características dos cimentos produzidos, enfatizou também a possível influência de sua adição na formação das fases hidratadas [1,6], reduzindo a energia de nucleação do C-S-H, melhorando o empacotamento do sistema nas idades iniciais $[12,20,50]$. Apesar do aumento da área superficial específica com a crescente adição de cinza de algaroba, os cimentos produzidos apresentaram área superficial específica inferior à apresentada pelo cimento padrão comercial. Esse fenômeno deu-se em função do processo de moagem do clínquer laboratorial ser menos refinado em relação ao comercial, apresentando grãos maiores (Tabela III) e consequentemente área superficial específica inferior. Salienta-se, no entanto, que a escolha da granulometria menos refinada para $\mathrm{C} 0 \mathrm{em}$ relação à do cimento comercial ocorreu para evidenciar a influência das adições propostas no processo de hidratação [31, 51]. Além disso, todos os cimentos $\mathrm{C} 0, \mathrm{C} 5, \mathrm{C} 10$ e $\mathrm{C} 15$ possuíram a mesma ordem de grandeza quanto à granulometria.

Os difratogramas das pastas hidratadas $(\mathrm{C} 0, \mathrm{CC} 5, \mathrm{CC} 10$, CC15 e CPII-F) aos 7 e 28 dias encontram-se expostos na Fig. 5. A adição de cinza de algaroba como material carbonático não alterou os compostos hidratados padrões para cimento Portland, comparativamente ao CPII-F, uma vez que foram identificados os principais compostos hidratados em sistemas cimentícios Portland, quais sejam (Fig. 5): C-S-H, portlandita, carbonato de etringita, bem como anidros de $\mathrm{C}_{3} \mathrm{~S}$ e $\mathrm{C}_{2} \mathrm{~S}$, identificados por meio das fichas: $\mathrm{Ca}(\mathrm{OH})_{2}$ (JCPDS 84-1263); $\mathrm{Ca}_{6} \mathrm{Al}_{2}\left(\mathrm{SO}_{4}, \mathrm{SiO}_{4}, \mathrm{CO}_{3}\right)_{3}(\mathrm{OH})_{12} 26 \mathrm{H}_{2} \mathrm{O}$ (JCPDS 41 1451); $\mathrm{C}_{3} \mathrm{~S}$ (JCPDS 41-1451); $\mathrm{C}_{2} \mathrm{~S}$ (JCPDS 33-0302); $\mathrm{CaCO}_{3}$

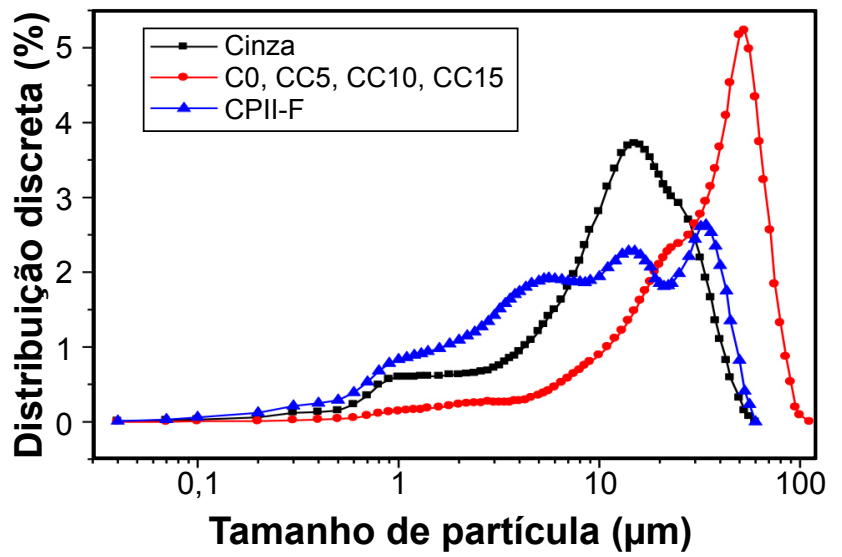

Figura 4: Curvas de distribuição granulométrica das matériasprimas.

[Figure 4: Particle size distribution curves of the raw materials.]

Tabela III - Distribuição granulométrica de partículas e características físicas dos cimentos estudados e da cinza de algaroba. [Table III - Particle size distribution and physical characteristics of the studied cements and the algaroba ash.]

\begin{tabular}{ccccccc}
\hline Material & $\begin{array}{c}\text { Diâmetro médio } \\
(\mu \mathrm{m})\end{array}$ & $\begin{array}{c}\text { D10 } \\
(\mu \mathrm{m})\end{array}$ & $\begin{array}{c}\text { D50 } \\
(\mu \mathrm{m})\end{array}$ & $\begin{array}{c}\mathrm{D} 90 \\
(\mu \mathrm{m})\end{array}$ & $\begin{array}{c}\text { Área superficial específica } \\
\left(\mathrm{m}^{2} / \mathrm{g}\right)\end{array}$ & $\begin{array}{c}\text { Densidade real } \\
\left(\mathrm{g} / \mathrm{cm}^{3}\right)\end{array}$ \\
\hline C0 & 32,19 & 6,30 & 30,82 & 59,37 & 0,914 & 3,045 \\
CC5 & 32,11 & 4,90 & 30,62 & 60,73 & 0,980 & 3,024 \\
CC10 & 29,25 & 4,28 & 27,24 & 56,88 & 0,982 & 3,012 \\
CC15 & 29,94 & 4,57 & 21,17 & 58,28 & 1,023 & 2,978 \\
CPII-F & 12,86 & 0,97 & 8,08 & 33,24 & 3,018 & 3,157 \\
Cinza & 13,75 & 1,50 & 11,92 & 28,67 & 4,400 & 2,552 \\
\hline
\end{tabular}


(JCPDS 12-1937); $\mathrm{Ca}_{15} \mathrm{SiO}_{35} \mathrm{xH}_{2} \mathrm{O}$ (JCPDS 12-1937); $\mathrm{Ca}_{2} \mathrm{SiO}_{4} 0,35 \mathrm{H}_{2} \mathrm{O}$ (JCPDS 33-0360); $\mathrm{Ca}_{4} \mathrm{Al}_{2} \mathrm{O}_{6} \mathrm{CO}_{3} 11 \mathrm{H}_{2} \mathrm{O}$ (JCPDS 41-0221); $\mathrm{Ca}_{4} \mathrm{Al}_{2} \mathrm{O}_{6}\left(\mathrm{CO}_{3}\right)_{05}(\mathrm{OH}) 11,5 \mathrm{H}_{2} \mathrm{O}$ (JCPDS 41-0219); $\mathrm{Ca}_{2} \mathrm{SiO}_{4} \times \mathrm{H}_{2} \mathrm{O}(\mathrm{JCPDS} 29-0373) ; \mathrm{Ca}_{3}\left[\mathrm{Si}_{3} \mathrm{O}_{8}(\mathrm{OH})_{2}\right]$ (JCPDS 83-1242); e MgO (JCPDS 077-2364). Em termos de $\mathrm{C}-\mathrm{S}$-H, alterações na cristalinidade no $\mathrm{Ca}_{1,5} \mathrm{SiO}_{3,5} \mathrm{xH}_{2} \mathrm{O}$ (pico 6, Fig. 5), $\mathrm{Ca}_{2} \mathrm{SiO}_{4} \mathrm{xH}_{2} \mathrm{O}$ (pico 9) e $\mathrm{Ca}_{3}\left[\mathrm{Si}_{3} \mathrm{O}_{8}(\mathrm{OH})_{2}\right]$ (pico 10) foram identificas, provavelmente causadas pela influência dos teores de cálcio, sílica e álcalis no sistema de hidratação [46, 47]. Como esperado, em termos de comportamento de um fíler calcário, as fases carboaluminatos [32, 36, 37] foram observadas no difratograma do cimento CPII-F (picos 2 e 7) aos 28 dias (Fig. 5), indicando que a metodologia de preparação, cura, paralisação da hidratação e análises dos sistemas hidratados foram coerentes. Salienta-se que tanto o monocarboaluminato (pico 8) quanto o hemicarboaluminato (pico 7) foram identificados quando da hidratação aos 28 dias do cimento comercial CPII-F.
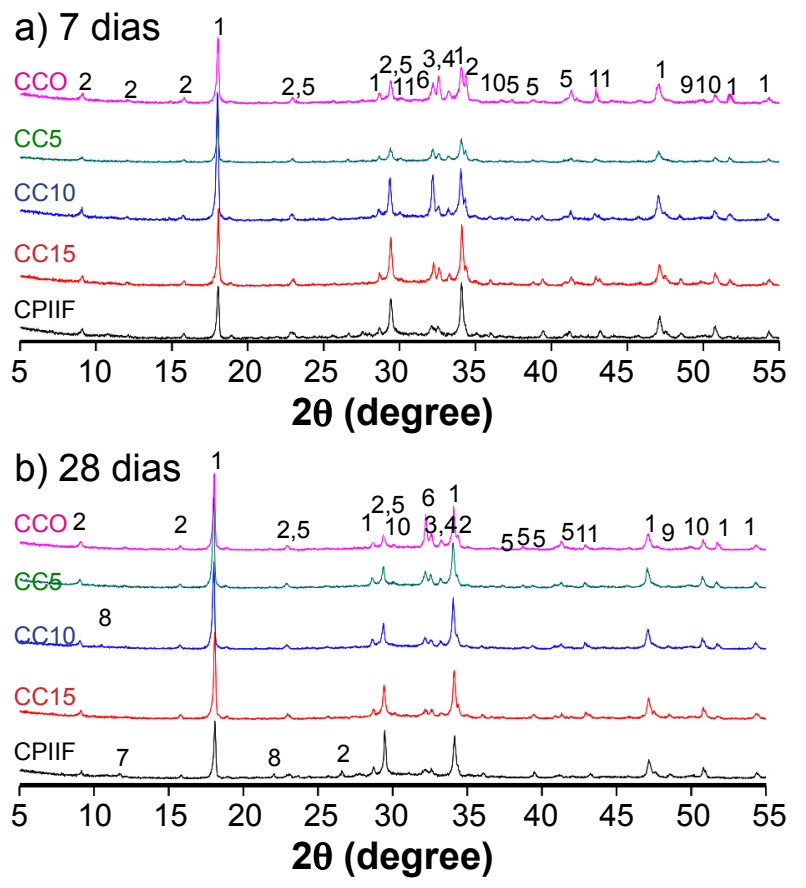

Figura 5: Difratogramas de raios X das pastas C0, CC5, CC10, CC15 e CPII-F hidratadas a 7 (a) e 28 (b) dias: $1-\mathrm{Ca}(\mathrm{OH})_{2}$; $2-\mathrm{Ca}_{6} \mathrm{Al}_{2}\left(\mathrm{SO}_{4}, \mathrm{SiO}_{4}, \mathrm{CO}_{3}\right)_{3}(\mathrm{OH})_{12} 26 \mathrm{H}_{2} \mathrm{O} ; 3-\mathrm{C}_{3} \mathrm{~S} ; 4-\mathrm{C}_{2} \mathrm{~S} ; 5-\mathrm{CaCO}_{3}$; $6-\mathrm{Ca}_{15} \mathrm{SiO}_{3,5} \mathrm{xH}_{2} \mathrm{O} ; 7-\mathrm{Ca}_{4} \mathrm{Al}_{2} \mathrm{O}_{6} \mathrm{CO}_{3} 11 \mathrm{H}_{2} \mathrm{O} ; 8 \mathrm{Ca}_{4} \mathrm{Al}_{2} \mathrm{O}_{6}\left(\mathrm{CO}_{3}\right)_{05}(\mathrm{OH}) 11,5 \mathrm{H}_{2} \mathrm{O}$; 9- $\mathrm{Ca}_{2} \mathrm{SiO}_{4} \mathrm{xH}_{2} \mathrm{O} ; 10-\mathrm{Ca}_{3}\left[\mathrm{Si}_{3} \mathrm{O}_{8}(\mathrm{OH})_{2}\right] ; \mathrm{e} 11-\mathrm{MgO}$.

[Figure 5: XRD patterns of the pastes CO, CC5, CC10, CC15 and CPII-F hydrated at 7 (a) and $28(b)$ days.]

Todavia, de uma forma geral, os cimentos laboratoriais com adição de cinza não apresentaram a fase carboaluminato (Fig. 5). Entretanto, constatou-se a presença do hemicarboaluminato, pico 8, para o cimento produzido CC10 aos 28 dias de cura (Fig. 6), o que sugeriu que a cinza de algaroba, por apresentar o carbonato de cálcio, favoreceu a formação dos carboaluminatos. Acredita-se que a falta de otimização de refino granulométrico dos grãos do clínquer para a produção desses cimentos laboratoriais tenha influenciado na reatividade de hidratação do $\mathrm{C}_{3} \mathrm{~A}$ (menos íons de $\mathrm{Al}_{2} \mathrm{O}_{3}$ disponíveis), modificando as condições de formação dos carboaluminatos, tendo em vista que a relação $\mathrm{Al}_{2} \mathrm{O}_{3} / \mathrm{SO}_{3}[41,52,53]$ e cinética de reação do calcário [1, $32,54]$ influenciam diretamente no sistema. Acredita-se que a variação na cinética de reação da formação das fases de hemicarboaluminato esteja diretamente relacionada com a lenta atividade química do calcário na hidratação $[37,55]$; todavia, a presença de potássio na sua forma hidratada acelera a reação do calcário, dando prioridade ao hemicarboaluminato em detrimento ao monocarboaluminato [53].

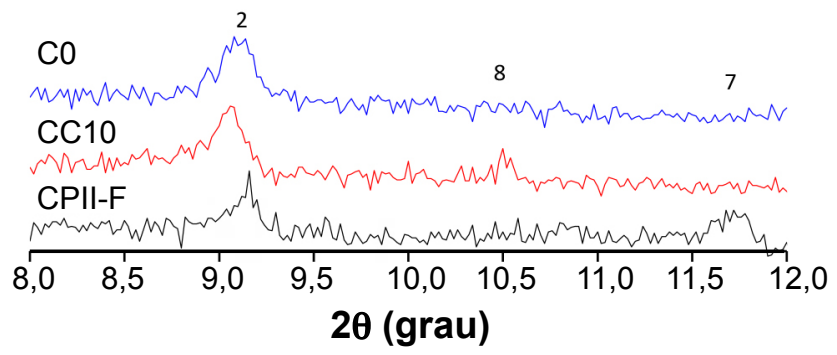

Figura 6: Difratogramas de raios $\mathrm{X}$ das pastas $\mathrm{C} 0, \mathrm{CC} 10$ e CPII-F hidratadas a 28 dias: $2-\mathrm{Ca}_{6} \mathrm{Al}_{2}\left(\mathrm{SO}_{4}, \mathrm{SiO}_{4}, \mathrm{CO}_{3}\right)_{3}(\mathrm{OH})_{12} 26 \mathrm{H}_{2} \mathrm{O}$; 7- $\mathrm{Ca}_{4} \mathrm{Al}_{2} \mathrm{O}_{6} \mathrm{CO}_{3} 11 \mathrm{H}_{2} \mathrm{O} ; 8-\mathrm{Ca}_{4} \mathrm{Al}_{2} \mathrm{O}_{6}\left(\mathrm{CO}_{3}\right)_{0,5}(\mathrm{OH}) 11,5 \mathrm{H}_{2} \mathrm{O}$.

[Figure 6: XRD patterns of the pastes CO, CC10 and CPII-F hydrated at 28 days.]

Os resultados das análises termogravimétricas, TG/ DTG, estão representados na Fig. 7, como também os valores das perdas de massa para diversas temperaturas na Tabela IV. Em 80, 145, 430 e $705{ }^{\circ} \mathrm{C}$, ocorreu a decomposição do C-S-H, etringita, monosulfato (AFm), $\mathrm{Ca}(\mathrm{OH})_{2}$ e carbonatos, respectivamente, corroborando a literatura [48]. A cinza de algaroba provocou alterações nos picos de C-S-H, tornando-os mais largos, com evidência no CC10 e CC15. Esse comportamento sugeriu a formação de uma estrutura química híbrida (C-S-H carbonatado) entre o carbonato de cálcio e o $\mathrm{C}-\mathrm{S}-\mathrm{H}$, picos próximos a $100{ }^{\circ} \mathrm{C}[1,32]$. No intervalo de 316 e $382{ }^{\circ} \mathrm{C}$ ocorreu outra decomposição do C-S-H, comportamento também observado por outros pesquisadores que utilizaram fíler de biomassa [56]. Na Fig. 8 estão apresentadas as micrografias obtidas por microscopia eletrônica de varredura (MEV) da pasta CC10. Pôde-se observar a presença de C-S-H carbonato, com morfologia característica, cujas agulhas apresentam um aspecto fundido [1, 57]. No intervalo de 300 a $350{ }^{\circ} \mathrm{C}$, o acréscimo da adição provocou maior perda de massa, proveniente da decomposição de $\mathrm{C}-\mathrm{S}-\mathrm{H}$, indicando que a presença da cinza melhorou a nucleação da fase C-S-H $[1,12,56]$. A decomposição da etringita ocorreu em temperaturas mais baixas na presença da cinza de algaroba, sendo preponderante em $\mathrm{CC} 10$ e $\mathrm{CC} 15$, em semelhança com o cimento comercial. A presença do carbonato de cálcio acelera a formação da etringita e prolonga a sua decomposição em monossulfato (AFm), proveniente da troca 
Tabela IV - Perdas de massa (\%) nas faixas de temperatura indicadas das pastas aos 28 dias de hidratação. [Table IV - Mass losses (\%) in the indicated temperature ranges of pastes at 28 days of hydration.]

\begin{tabular}{cccccc}
\hline Pasta & $30-140{ }^{\circ} \mathrm{C}$ & $140-410{ }^{\circ} \mathrm{C}$ & $410-530{ }^{\circ} \mathrm{C}$ & $530-760{ }^{\circ} \mathrm{C}$ & $760-900{ }^{\circ} \mathrm{C}$ \\
\hline $\mathrm{C} 0$ & 5,45 & 3,27 & 3,03 & 3,73 & 0,36 \\
$\mathrm{CC} 5$ & 6,08 & 3,76 & 3,76 & 8,35 & 0,43 \\
$\mathrm{CC} 10$ & 5,97 & 3,97 & 3,41 & 11,50 & 0,50 \\
$\mathrm{CC} 15$ & 6,03 & 3,50 & 3,51 & 13,44 & 0,35 \\
$\mathrm{CPII}-\mathrm{F}$ & 5,90 & 3,00 & 2,70 & 15,08 & 0,59 \\
\hline
\end{tabular}

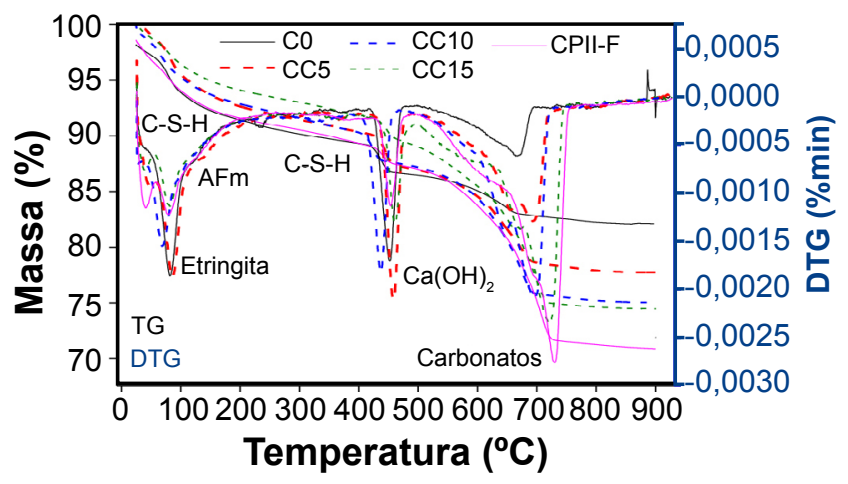

Figura 7: Curvas termogravimétricas TG e DTG das pastas $\mathrm{C} 0$, CC5, CC10, CC15 e CPII-F hidratadas aos 28 dias.

[Figure 7: TG and DTG thermogravimetric curves of the pastes C0, CC5, CC10, CC15 and CPII-F hydrated at 28 days.]
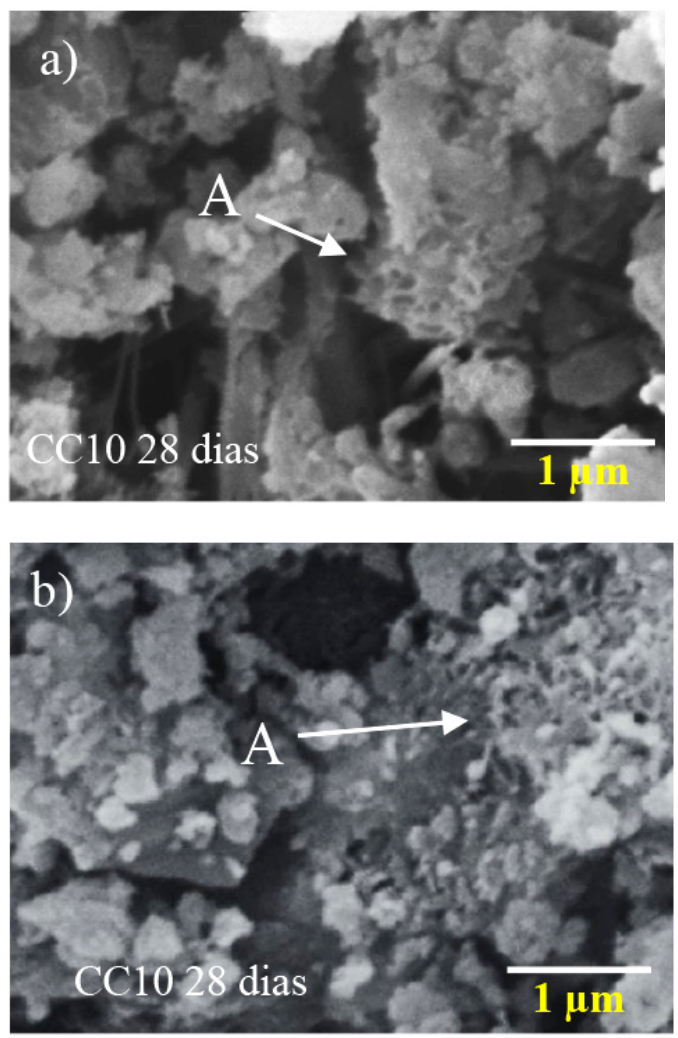

Figura 8: Micrografias obtidas por MEV da pasta CC10 hidratada aos 28 dias: A- C-S-H carbonatado.

[Figure 8: SEM micrographs of the paste CC10 hydrated at 28 days: A- carbonated $\mathrm{C}-\mathrm{S}-\mathrm{H}$.] de íons de sulfato por íons de carbonato na hidratação do $\mathrm{C}_{3} \mathrm{~A}$ [32, 36, 37]. A fase monossulfato (AFm) mostrou alteração na temperatura de decomposição com a incorporação da cinza de algaroba, onde os picos endotérmicos ficaram cada vez mais sutis, possivelmente pela interferência das finas partículas de $\mathrm{CaCO}_{3}$, que, reagindo com o $\mathrm{C}_{3} \mathrm{~A}$, formaram $\mathrm{C}_{3} \mathrm{ACaCO}_{3} 11 \mathrm{H}_{2} \mathrm{O}[32,33]$. Por fim, a liberação de dióxido de carbono foi evidenciada pela formação de dois picos endotérmicos entre 620 e $710^{\circ} \mathrm{C}$, ligada à carbonatação do $\mathrm{Ca}(\mathrm{OH})_{2}$ e à decomposição do $\mathrm{CaCO}_{3}$ adicionado através da cinza (Fig. 7).

\section{CONCLUSÕES}

Quanto às características físico-químicas, a cinza de algaroba (Prosopis juliflora) demonstrou ser um material carbonático promissor para uso como fíler calcário em materiais cimentícios, uma vez que apresentou predominância de compostos com cálcio, $\mathrm{CaO}$, teor de álcalis dentro do padrão, granulometria refinada, alta área superficial específica, baixa densidade e ausência de óxidos expansivos. Em termos de reatividade química no sistema cimentício, a cinza de algaroba evidenciou o potencial como fíler calcário alternativo, uma vez que foram identificados os compostos hidratados padrões para cimento Portland nos sistemas cimentícios com adição da cinza, atuando inclusive na influência da formação da fase carboaluminato. Por fim, observou-se a estabilização da etringita e o aumento da formação da fase C-S-H.

\section{REFERÊNCIAS}

[1] V. Sklivaniti, P. Tsakiridis, N. Katsiotis, D. Velissariou, N. Pistofidis, D. Papageorgiou, M. Beazi, J. Environ. Chem. Eng. 5 (2017) 205.

[2] J. Torkaman, A. Ashori, A.S. Momtazi, Constr. Build. Mater. 50 (2014) 432.

[3] S. Maschio, G. Tonello, L. Piani, E. Furlani, Chemosphere 85 (2011) 666.

[4] S.M.S. Kazmi, M.J. Munir, I. Patnaikuni, Y.-F. Wu, Constr. Build. Mater. 148 (2017) 231.

[5] K. Kupwade-Patil, A.F. Al-Aibani, M.F. Abdulsalam, C. Mao, A. Bumajdad, S.D. Palkovic, O. Büyüköztürk, Constr. Build. Mater. 113 (2016) 423.

[6] E. Berodier, K. Scrivener, J. Am. Ceram. Soc. 97 (2014) 
3764.

[7] R. Pilar, R.A. Schankoski, A.J. Dal Moro, W.L. Repette, Matéria 21 (2016) 92-104.

[8] EN 14216, "Cement, composition, specifications and conformity criteria for common cements", BSI, London (2011).

[9] ASTM C150, "Standard specification for Portland cement" (2012).

[10] J. Rosales, M. Cabrera, M.G. Beltrán, M. López, F. Agrela, J. Clean. Prod. 154 (2017) 424.

[11] D. Panesar, M. Aqel, D. Rhead, H. Schell, Cem. Concr. Res. 80 (2017) 175.

[12] G.D. Moon, S. Oh, S.H. Jung, Y.C. Choi, Constr. Build. Mater. 135 (2017) 129.

[13] S. Mayooran, S. Ragavan, N. Sathiparan, J. Build. Eng. 13 (2017) 137.

[14] S. Abbas, S.M.S. Kazmi, M.J. Munir, Constr. Build. Mater. 132 (2017) 61.

[15] C. Fapohunda, B. Akinbile, A. Shittu, Int. J. Sustain. Built Environ. 6 (2017) 675.

[16] O. Benjeddou, C. Soussi, M. Jedidi, M. Benali, J. Build. Eng. 10 (2017) 32.

[17] ABNT NBR 11578, "Cimento Portland composto especificação”, Ass. Bras. Normas Técn. (1991).

[18] ABNT NBR 5733, "Cimento Portland de alta resistência inicial”, Ass. Bras. Normas Técn. (1991).

[19] ABNT NBR 5736, "Cimento Portland pozolânico", Ass. Bras. Normas Técn. (1991).

[20] W. Deboucha, N. Leklou, A. Khelidj, M.N. Oudjit, Constr. Build. Mater. 146 (2017) 687.

[21] E.M.J. Berodier, "Impact of the supplementary cementitious materials on the kinetics and microstructural development of cement hydration", Thèse, École Polytech. Féd. Lausanne, Lausanne (2015).

[22] K.L. Scrivener, P. Juilland, P.J.M. Monteiro, Cem. Concr. Res. 78 (2015) 38.

[23] R. Kumar, N. Chandrashekar, J. Ind. Acad. Wood Sci. 13 (2016) 101.

[24] C.C. Ban, M. Ramli, Int. J. Phys. Sci. 6 (2011) 6596.

[25] C.B. Cheah, M. Ramli, Constr. Build. Mater. 30 (2012) 320.

[26] R. Siddique, Resour. Conserv. Recycl. 67 (2012) 27.

[27] C.B. Cheah, M. Ramli, Compos. Part B-Eng. 51 (2013) 68.

[28] R.P. Girón, B. Ruiz, E. Fuente, R.R. Gil, I. Suárez-Ruiz, Fuel 114 (2013) 71.

[29] R.C.E. Modolo, V.M. Ferreira, L.A. Tarelho, J.A. Labrincha, L. Senff, L. Silva, Constr. Build. Mater. 45 (2013) 275.

[30] F. Matalkah, P. Soroushian, S. Ul Abideen, A. Peyvandi, Constr. Build. Mater. 121 (2016) 491.

[31] W. Huang, H. Kazemi-Kamyab, W. Sun, K. Scrivener, Cem. Concr. Compos. 77 (2017) 86.

[32] V.L. Bonavetti, V.F. Rahhal, E.F. Irassar, Cem. Concr.
Res. 31 (2001) 853.

[33] W. Al-Kutti, A.S. Islam, M. Nasir, J. King Saud Univ. Eng. Sci. (2017).

[34] A. Bazzoni, S. Ma, Q. Wang, X. Shen, M. Cantoni, K.L. Scrivener, J. Am. Ceram. Soc. 97 (2014) 3684.

[35] A. Schöler, B. Lothenbach, F. Winnefeld, M.B. Haha, M. Zajac, H.-M. Ludwig, Cem. Concr. Res. 93 (2017).

[36] B. Dilnesa, B. Lothenbach, G. Le Saout, G. Renaudin, A. Mesbah, Y. Filinchuk, A. Wichser, E. Wieland, Cem. Concr. Res. 41 (2011) 311.

[37] S.Adu-Amankwah, M.Zajac, C. Stabler, B.Lothenbach, L. Black, Cem. Concr. Res. 100 (2017) 96.

[38] P. Hewlett, Lea's chemistry of cement and concrete, Butterworth-Heinemann (2003).

[39] V. Tydlitát, T. Matas, R. Černý, Constr. Build. Mater. 50 (2014) 140 .

[40] J. Cuenca, J. Rodríguez, M. Martín-Morales, Z. Sánchez-Roldán, M. Zamorano, Constr. Build. Mater. 40 (2013) 702.

[41] F. Deschner, F. Winnefeld, B. Lothenbach, S. Seufert, P. Schwesig, S. Dittrich, F. Goetz-Neunhoeffer, J. Neubauer, Cem. Concr. Res. 42 (2012) 1389.

[42] M. Singh, S. Upadhayay, P. Prasad, Waste Manage. 16 (1996) 665.

[43] M. Singh, S. Upadhayay, P. Prasad, Cem. Concr. Res. 27 (1997) 1037.

[44] E. Gartner, T. Sui, Cem. Concr. Res. (2017).

[45] L. Simão, J. Jiusti, N.J. Lóh, D. Hotza, F. RauppPereira, J.A. Labrincha, O.R.K. Montedo, Process Saf. Environ. Prot. 109 (2017) 106.

[46] B. Lothenbach, A. Nonat, Cem. Concr. Res. 78 (2015) 57.

[47] J. Haas, A. Nonat, Cem. Concr. Res. 68 (2015) 124.

[48] H.F. Taylor, Cement chemistry, Thomas Telford (1997).

[49] B. Ilić, V. Radonjanin, M. Malešev, M. Zdujić, A. Mitrović, Constr. Build. Mater. 133 (2017) 243.

[50] L. Nicoleau, Transp. Res. Record 2142 (2010) 42.

[51] C. Çetin, S.T. Erdoğan, M. Tokyay, Cem. Concr. Compos. 67 (2016) 39.

[52] M. Boháč, M. Palou, R. Novotný, J. Másilko, D. Všianský, T. Staněk, Constr. Build. Mater. 64 (2014) 333.

[53] M. Zajac, A. Rossberg, G. Le Saout, B. Lothenbach, Cem. Concr. Compos. 46 (2014) 99.

[54] D.K. Panesar, M. Aqel, D. Rhead, H. Schell, Cem. Concr. Compos. 80 (2017) 175.

[55] K. De Weerdt, M.B. Haha, G. Le Saout, K.O. Kjellsen, H. Justnes, B. Lothenbach, Cem. Concr. Res. 41 (2011) 279. [56] E.Y. Nakanishi, M. Frías, S.F. Santos, M.S. Rodrigues, R. Vigil de la Villa, O. Rodriguez, H.S. Junior, J. Clean. Prod. 116 (2016) 236.

[57] A. Morandeau, M. Thiéry, P. Dangla, Cem. Concr. Res. 56 (2014) 153.

(Rec. 31/10/2017, Rev. 04/05/2018, 11/07/2018, Ac. 10/09/2018) 